\title{
Role of Diffusion Mri In Differentiation Between The Common Pediatric Posterior Fossa Brain Tumors.
}

\author{
HanaaAbdelkader Ahmed ${ }^{\mathrm{a}}$, Eman A. F. Darwish ${ }^{\mathrm{a}}$, Osama Mohamed abo-bakrkhattab
}

a. Radiology Department, Faculty of Medicine, Ain Shams University.

b. Radiology Department, Basioun Central Hospital,Basioun, Gharbia.

Corresponding author: Osama Mohamed abo-bakrkhattab,Tel: +20 1065448747, E-Mail: Khattabosama@yahoo.com

\section{Abstract}

Background: Diffusion weighted imaging (DWI) and apparent diffusion coefficient (ADC) map provide information on MRI about the cellularity of the tumor and have an important role in the pre-operative differentiation of different tumor types.

Objective: The aim of this work is to assess the role of diffusion MRI in differentiation between the most frequently encountered pediatric posterior fossa brain tumors.

Materials and methods: Twenty-four patients were prospectively included in this study. They were referred from the Neurosurgery Department. All of them were suspected to have posterior fossa SOL according to the contrast enhanced CT. All patients were subjected to conventional MRI followed by diffusion MR imaging and calculation of the ADC values.

Results: Twenty-four children (15 females) were included in the study. Their ages ranged between one and fifteen years old with a mean age of six years. Low-and high-grade tumors could be differentiated by using both absolute ADC values and ratios. Low-grade tumors showed statistically significantly higher ADC values (1.69 \pm 0.15 vs. $0.80 \pm 0.23)$ and ratios for tumor versus normal cerebellum $(2.17 \pm 0.30$ vs. $1.14 \pm 0.33)$ and tumor versus brain stem $(1.88 \pm 0.35$ vs. $1.06 \pm 0.24)$. The probability of error at 0.05 was considered significant, while at 0.01 and 0.001 was considered highly significant. Absolute ADC values and cerebellar and brainstem ratios were significantly higher in low-grade astrocytomas than in MBs. Overlap was found between ADC values of ATRTs and MBs.The sensitivity and specificity of a cutoff ADC value of $>1.083 \times 10^{-3} \mathrm{~mm}^{2} / \mathrm{s}$ for differentiation of pilocytic astrocytomas from MBs and ependymomas were 100\%. The sensitivity and specificity of a cutoff ADC value of $\leq 0.847 \times 10^{-3} \mathrm{~mm}^{2} / \mathrm{s}$ for differentiation of medulloblastomas from PAs and ependymomas were $100 \%$. The sensitivity and specificity of a cutoff ADC value of $\leq 1.083$ x $10^{-3} \mathrm{~mm}^{2} / \mathrm{s}$ and $>0.847 \times 10^{-3} \mathrm{~mm}^{2} / \mathrm{s}$ for ependymomas were $100 \%$.

Conclusion: The calculation of ADC value in the solid enhancing portion of a tumor is a simple and reliable technique for preoperative differentiation of the most common posterior fossa tumors.

Keywords:DWI,ADC,Posterior fossa tumors.

Introduction

Brain tumors are the most common solid tumors in childhood and the second most common neoplasm in childhood after hematological malignancies. However, they are the leading cause of morbidity and mortality. $\mathrm{Up}$ to $60-70 \%$ of brain tumors are infra tentorial, being most common in children from 4 to 11 years $^{[1]}$. Common posterior fossa brain tumors in children include juvenile pilocytic astrocytoma (JPA), medulloblastoma (MB), ependymoma and brainstem gliomas. Because these various tumors require very different treatment approaches and have significantly different natural histories and outcomes, an accurate and specific diagnosis is mandatory ${ }^{[1]}$.
Histopathological evaluation of brain biopsies is still the gold standard for definitive diagnosis. However, there are also limitations to histological diagnosis e.g. sampling errors in surgical biopsies due to intrinsic tissue heterogeneity where tumors under grading can occur. Also, due to the complication of being invasive. So the development of new noninvasive diagnostic tools is necessary ${ }^{[1]}$.

Diffusion MR imaging is a technique in which dedicated phase-defocusing and refocusing gradients allow evaluation of microscopic water diffusion within tissues. It has been considered a means to characterize and differentiate morphologic features, including edema, necrosis, and tumor tissue, by measuring differences in apparent diffusion 
coefficient (ADC) caused by water proton mobility alterations. Recent studies evaluated the role of diffusion-weighted imaging (DWI) in differentiating type and grade of pediatric brain tumors in the posterior fossa with promising results ${ }^{[2]}$.

\section{Patients and methods}

The study included 24 patients from the pediatric population with posterior fossa tumors (as proved by a radiological report) referred from Ain Shams Pediatric Hospital or Neurosurgery Department at Ain ShamsUniversity to our department. The study was approved by the Ethics Board of Ain Shams University and an informed written consent was taken from each participant in the study.

Inclusion criteria: included pediatric age group (1 day to 18 years old) and patient with radiological evidence of presence of posterior fossa neoplasm.

Exclusion criteria: included patients over 18 years old, extra axial masses, purely cystic masses, patients known to have contraindications for MRI, e.g. an implanted magnetic device and pacemakers and patients with contraindication to contrast media e.g. elevated renal function tests.

Patient preparation included detailed explanation of the procedure to the patient (if he was old enough) or the patient's parents.Obtaining informed consent from the parents and attending anesthesiologist to be responsible for evaluation of renal function and administration of contrast media according to body weight provided renal function was normal.In addition, administration of sedation and any preparation required if the child was unable to stay stationary throughout the procedure.

\section{Technique and MR imaging data acquisition:}

\section{Conventional MRI:}

The study was performed on 1.5 Tesla MRI machine using head coil with patient lying in the supine position.

All patients underwent routine MR brain imaging, including:

- Sagittal and axial T1-wieghted non-contrast images with imaging parameters of 538/10 (TR/TE).
- Axial fast spin-echo T2-weighted, T2weighted spin-echo images with imaging parameters of 4130/120 (TR/TE).

- Axial fluid-attenuated inversion recovery (FLAIR) with imaging parameters of 11000/140 (TR/TE).

- Post contrast enhanced axial, coronal, and sagittal T1-weighted images.

\section{Diffusion weighted MR imaging (DWI):}

The imaging sequence for DWI was a multi-section single shot spin echo EPI sequence (TR/TE/NEX: $3704 / 88 \mathrm{~ms} / \mathrm{l}$ ) with diffusion sensitivities of $\mathrm{b}$ values $=0$ and $1000 \mathrm{~s} / \mathrm{mm}^{2}$. The diffusion gradients were applied sequentially in three orthogonal directions (X, Y \& Z directions). Sections of $5 \mathrm{~mm}$ thickness, interstice gap of $1 \mathrm{~mm}$, FOV $163 \mathrm{~mm}$ and a matrix of $128 \cdot 256$ was used for all images.

The ADC maps were generated automatically by MRI software and included in the sequences.

\section{Image analysis and results:}

T2, T1, and post contrast weighted imageswere analyzed for localization of solid non-necrotic, non-hemorrhagic, noncalcified and enhancing part of the tumors distinct from peritumoral edema.

Once identified, this area was qualitatively assessed for presence or absence of restricted diffusion on DWIs and corresponding ADC map.

Restricted diffusion is defined as an area of high signal intensity on (b value 1000) images and a corresponding low ADC values (a dark area on ADC map).

For quantitative analysis 3 different regions of interest were placed within the identified region on consecutive slices on the ADC map. If the enhancing solid portion of the tumor was small ROIs were placed within the same slice but care was taken to ensure that the ROIs didn't overlap. The ADC value for each ROI was obtained and then averaged to serve as the tumor average ADC value.

An additional region of interest was placed within normal appearing cerebellar/ brain stem (if tumor is located within the brainstem) tissue to obtain a control ADC value. 
The ratio of average tumor ADC to the normal ADC was calculated.

This along with the average tumor ADC value was compared to the histopathological results of post-operative specimens which specified tumor diagnosis, histological subtypes if relevant and $\mathrm{WHO}$ grading.

If applicable, the best ADC cut off values that allow for differentiation between different histological types and grades of commonly encountered posterior fossa tumors was obtained.

\section{Statistical Analysis}

ADC values and ratios of JPAs, medulloblastomas, ependymomas, ATRTs and DIPGs were compared by using a 2-tailed t test andone-way analysis of variance (ANOVA).The observed differences were considered statistically significant if $\mathrm{p}$ value at 0.05 , while at 0.01 and 0.001 was considered highly significant.

\section{Results}

The current study included a cohort of twenty five children with posterior fossa tumors but one patient was excluded as he had extensive calcific tumor that did not have interpretable ADC. They were nine males and fifteen females. Their ages ranged between one and fifteen years with a mean age of six years.

According to histological examination, there were nine patients with medulloblastoma(MB)(WHO grade IV), six patients with pilocytic astrocytoma (PA)(WHO grade I),two patients with ependymoma(EPN)(WHO grade III), two patients with atypical teratoidrhabdoid tumor(ATRT)(WHO grade IV). Additionally there were five cases of high grade diffuse intrinsic pontine glioma (DPG) which were diagnosed on the basis of conventional MRI findings as histopathological confirmation was not obtained in these patients owing to the difficulty of obtaining a biopsy specimen from the brainstem.

Overall, eighteen tumors $(75 \%)$ were classified as highgrade, six $(25 \%)$ as lowgrade.

Low- and high-grade tumors could be differentiated by using both absolute ADC values and ratios. Lowgradetumors showed statistically significantly higher ADC values $(\mathbf{1 . 6 9} \pm \mathbf{0 . 1 5}$ vs. $\mathbf{0 . 8 0} \pm \mathbf{0 . 2 3})$ and ratios for tumorversus normal cerebellum $(2.17 \pm 0.30$ vs. $1.14 \pm 0.33)$ and tumor versus brain stem $(\mathbf{1 . 8 8} \pm \mathbf{0 . 3 5}$ vs. $\mathbf{1 . 0 6} \pm \mathbf{0 . 2 4})$. The probability of error at 0.05 was considered significant, while at 0.01 and 0.001 was considered highly significant as shown in table (1).The sensitivity and specificity of a cutoff ADC value of $>1.327 \times 10^{-3} \mathrm{~mm}^{2} / \mathrm{s}$ for differentiation of low grade from high grade tumors were $100 \%$.

Table (1): Summary of ADC values, ADC ratios of tumors to normal appearing cerebellum, and ADC ratios of tumors to brainstem forlow-grade and high grade tumors

\begin{tabular}{|c|c|c|c|c|}
\hline & $\begin{array}{l}\text { Low-grade tumors } \\
(n=6 ; \text { mean } \pm S D\end{array}$ & \begin{tabular}{|l|} 
High-grade tumors \\
$(n=18 ;$ mean $\leq S D$
\end{tabular} & $P$-value & Sig. \\
\hline Mean ADC values $(10-3 \mathrm{~mm} 2 / \mathrm{s})$ & $1.69056 \pm 0.15$ & $0.80352 \pm 0.23$ & 0 & HS \\
\hline ADC ratio (tumornormal cerebellum) & $2.17858 \pm 0.30$ & $1.14403 \pm 0.33$ & 0 & HS \\
\hline ADC ratio (tumorn normal brain stem) & $1.87921 \pm 0.35$ & $1.05526 \pm 0.24$ & 0.001 & HS \\
\hline
\end{tabular}

Pilocytic astrocytomas and MBs could also be differentiated by using both ADC values as well as the tumor/cerebellum and tumor/brainstem ratios. Pilocytic astrocytomas showed significantly higher ADC values (1.69056 $\pm 0.15 \mathrm{vs}$. $0.69963 \pm 0.12, \quad \boldsymbol{P}=\mathbf{0})$ as well as tumor/cerebellum $\quad(\mathbf{2 . 1 7 8 5 8} \pm \mathbf{0 . 3 0} \mathrm{vs}$. $0.95678 \pm 0.21, \quad P=0$ ) and tumor/brainstem ratios

$0.93709 \pm 0.16, P=0.001)$ compared to MBs.

Ependymomas showed significantly lower ADC values (1.03333 $\pm 0.07 \mathrm{vs}$. $1.69056 \pm 0.15, \boldsymbol{P}=\mathbf{0 . 0 0 1})$ as well as tumor/cerebellum $2.17858 \pm 0.30, \boldsymbol{P}=\mathbf{0 . 0 0 1})$ ratios $\quad(1.21644 \pm 0.02 \mathrm{vs}$. $1.87921 \pm 0.35, \boldsymbol{P}=\mathbf{0 . 0 0 5}) \quad$ compared to Pilocytic astrocytomas.

ADC values $\quad(1.03333 \pm 0.07 \mathrm{vs}$. $0.69963 \pm 0.12, \boldsymbol{P}=\mathbf{0 . 0 2})$ as well as tumor/ cerebellum $(1.36121 \pm 0.03 \mathrm{vs}$. $0.95678 \pm 0.21, P=0$ ) and tumor/ brainstem ratios $\quad(1.21644 \pm 0.02$ vs. $\quad \mathbf{0 . 9 3 7 0 9} \pm \mathbf{0 . 1 6}$, $\mathrm{P}=0.001$ ) of ependymomas were significantly higher compared toMBs. 
Pilocytic astrocytomas and ATRTcould be differentiated by using both ADC values as well as the tumor/cerebellum and tumor/brainstem ratios. Pilocytic astrocytomas showed significantly higher ADC values (1.69056 $\pm 0.15 \mathrm{vs}$. $0.685 \pm 0.002, \quad \boldsymbol{P}=\mathbf{0})$ as well as tumor/cerebellum $\quad(\mathbf{2 . 1 7 8 5 8} \pm \mathbf{0 . 3 0} \mathrm{vs}$. $\mathbf{0 . 8 6 7 2} \pm 0.01, \quad P=0)$ and tumor/brainstem ratios

$0.80597 \pm 0.01, P=0.001)$ $(\mathbf{1 . 8 7 9 2 1} \pm 0.35$ vs. ATRTs.

ATRTs and ependymomas could also be differentiated by using both ADC values as well as the tumor/cerebellum and tumor/brainstemratios. ATRT showed significantly lower ADC values $(0.685 \pm 0.002$ vs. $1.0333 \pm 0.07, \boldsymbol{P}=\mathbf{0 . 0 5})$ as well as tumor/cerebellum $(\mathbf{0 . 8 6 7 2} \pm \mathbf{0 . 0 1} v s$. $1.36121 \pm 0.03, \quad P=0.016) \quad$ and tumor/brainstem ratios $\quad(\mathbf{0 . 8 0 5 9 7} \pm \mathbf{0 . 0 1} v \mathrm{vs}$. $1.21644 \pm 0.004, P=0.004) \quad$ compared to ependymomas.

However,ATRTs and MBscould not be differentiated by using ADC values and there was any statistical significant difference.

There was no overlap between ADC values and ratios of Pilocytic astrocytomas and MBs, ependymomas andpilocytic astrocytomas as well as for of Pilocytic astrocytomasand ATRT, respectively.

Also, there was no overlap between ADC values and ratios ofMB and Ependymoma as well as for ATRTsand ependymomas but overlap was found between ADC values of ATRTs and MBs.

- The sensitivity and specificity of a cutoff ADC value of $>\mathbf{1 . 0 8 3} \times \mathbf{1 0}^{-}$ ${ }^{3} \mathbf{m m}^{2} / \mathrm{s}$ for differentiation of pilocytic astrocytomas from MBs and ependymomas were $100 \%$.

- The sensitivity and specificity of a cutoff $\mathrm{ADC}$ value of $\leq \mathbf{0 . 8 4 7} \times \mathbf{1 0}^{-}$ ${ }^{3} \mathrm{~mm}^{2} / \mathrm{s}$ for differentiation of medulloblastomas from PAs and ependymomas were $100 \%$.

- The sensitivity and specificity of a cutoff ADC value of $\leq \mathbf{1 . 0 8 3 x} \mathbf{1 0}^{-}$
${ }^{3} \mathrm{~mm}^{2} / \mathrm{s}$ and $>0.847 \quad \times \quad 10^{-3} \mathrm{~mm}^{2} / \mathrm{s}$ for ependymomas were $100 \%$.

ADC values as well as tumor/ cerebellum and tumor/ brainstem ratios of DPGs were compared to the rest of the tumors encountered in our study and revealed that PAs and DPGs could be differentiated by using both ADC values as well as the tumor/cerebellum and tumor/brainstem ratios. DPGs showed significantly lower ADC values as well as tumor/cerebellum and tumor/brainstem ratios compared to PAs. But when DPGs were compared to MBs, Ependymomas and ATRTs respectively, could not be differentiated by using ADC values and there was any statistical significant difference.

\section{Discussion}

Pediatric central nervous system (CNS) tumors encompass a heterogeneous group of cell types. Clinical treatment and prognosis varies with tumor type, grade, stage, and extent of resection ${ }^{[3]}$.

Diffusion-weighted (DW) imaging can provide information about tumor cellularity, thereby helping in the characterization and grading of brain tumors ${ }^{[4]}$.

DWI assesses water diffusivity within intra- and extra-cellular spaces by means of apparent diffusion coefficient (ADC) measurements. Visual inspection of diffusion and ADC images has been reported as not very useful in differentiating between tumor types, where as an important trend has appeared toward the use of quantitative diffusion imaging technique ${ }^{[5]}$.

In this study, we prospectively evaluated the role of DW MRI and ADC values in characterizing and differentiating common posterior fossa tumors in pediatric patients. Results were correlated to post-operative pathological findings.

This study included twenty four patients with posterior fossa tumors. Age ranged between 1 year and 15 years old. The mean age of the cases was 6 years.

Rumboldt et al. $^{[6]}$ included thirty-one patients with neuroglial tumors and the mean age of the patients was 10 years (range, 6 months to 16 years old). The study group in this work encompassed the 
most common posterior fossa tumors in children; six cases of juvenile pilocytic astrocytoma (JPA), nine cases of medulloblastoma (MB) and two cases of ependymoma. It also included other less common posterior fossa tumors ${ }^{[6]}$.It has been reported that high-grade gliomas typically are hyperintense on DW images with decreased ADC values. High tumor cellularity is probably the major determinant of the decreased ADC values in high-grade brain tumors ${ }^{[7]}$.

Other studies have suggested that ADC correlates not only with tumor cellularity, but also with total nuclear area and tumor grade with high-grade tumors having high cellular density and decreased ADC ${ }^{[8]}$.

In our study, results showed a significant difference between ADC in different histological subgroups. ADC values were significantly different between the commonest three posterior fossa tumors in children: pilocytic astrocytomas, ependymomas and medulloblastomas.

Low water diffusion observed in pilocytic astrocytomas probably reflects the low cellularity appearance of these tumors on histologic examination ${ }^{[6]}$.ADC values showed high significant difference between pilocytic astrocytomas $\left(1.69056 \pm 0.15 \times 10^{-3} \mathrm{~mm}^{2} / \mathrm{s}\right) \quad$ (mean $\pm \mathrm{SD}$ ) and medulloblastomas $\left(0.69963 \pm 0.12 \mathrm{x}^{-}\right.$ ${ }^{3} \mathrm{~mm}^{2} / \mathrm{s}$ ) with a $\mathrm{P}$ value $<0.01$.

The study also demonstrated significant difference between pilocytic astrocytomas $\left(\begin{array}{lll}\left.1.69056 \pm 0.15 \quad \times 10^{-3} \mathrm{~mm}^{2} / \mathrm{s}\right) & \text { and }\end{array}\right.$ ependymomas $\quad\left(1.03333 \pm 0.07 \quad \mathrm{x} 10^{-}\right.$ ${ }^{3} \mathrm{~mm}^{2} / \mathrm{s}$ ). Calculated $\mathrm{P}$ value was $\leq 0.005$.

We found a significant difference when comparing $(0.69963 \pm 0.12$ medulloblastomas $\left.\mathrm{x} 10^{-3} \mathrm{~mm}^{2} / \mathrm{s}\right) \quad$ with ependymomas $\quad\left(1.03333 \pm 0.07 \quad \mathrm{x} 10^{-}\right.$ ${ }^{3} \mathrm{~mm}^{2} / \mathrm{s}$ ), with a $\mathrm{P}$ value $<0.05$. These results coincide with that of Kan et al. ${ }^{[9]}$ where the preoperative DWI and apparent diffusion coefficient (ADC) characteristics of brain tumors in 41 children were compared with histologic diagnosis. Signal characteristics on DWI and ADC maps correlated well with tumor grade.
Rumboldt et al. ${ }^{[6]}$ found that ADC values were significantly higher in pilocytic astrocytomas $(1.65 \pm 0.27) \quad\left(\times 10^{-3} \mathrm{~mm}^{2} / \mathrm{s}\right)$ $($ mean \pm SD) than in ependymomas $(1.10 \pm$ $0.11)(\mathrm{P}=.0003)$ and medulloblastomas $(0.66 \pm 0.15) \quad\left(\mathrm{x} 10^{-3} \mathrm{~mm}^{2} / \mathrm{s}\right) \quad(\mathrm{P}<.0001)$. Ependymomas demonstrated significantly higher ADC values than medulloblastomas $(\mathrm{P}=0.0005)$. The range of ADC values and ratios within JPAs and ependymomas did not overlap with that of medulloblastomas.Rumboldt et al. ${ }^{[6]}$ also, stated that JPA and medulloblastoma could be differentiated on the basis of ADC values and ADC ratios in all patients, and there was no overlap in obtained measurements between these two tumor types.

ADC values and ratios also clearly distinguished medulloblastoma from ependymoma in all patients, again without any overlap. This finding is similar to that in a recent study by Yamasaki et al. [10] who found that ADC values were retrospectively $100 \%$ accurate in differentiation between ependymomas and PNETs.Similar findings deduced from our study showed no significant overlap between the ADC of low-grade astrocytoma and ependymoma or medulloblastoma and ependymoma.

Schneider et al. ${ }^{[11]}$ found that ADC of JPA and medulloblastoma (whether minimum or mean) did overlap.The ADC values of JPAs were also higher from ependymomas in their study, and this difference was statistically significant though there was a slight overlap in measurements, which corresponds to results reported by Yamasaki et al. ${ }^{[10]}$.

Medulloblastomas and AT/RTs may be indistinguishable by their diffusion characteristics on MR imaging ${ }^{[6]}$. These findings in JPAs and medulloblastomas are probably secondary to the low cellularity and relatively small nuclear area typically seen in the former tumor types in contradistinction to the densely packed cells and large nuclei characteristic for the latter ${ }^{[12,13]}$.

ADC value of AT/RT tumors in our study were obtained in two cases and were as follows: 0.68 and $0.69\left(\times 10^{-3} \mathrm{~mm}^{2} / \mathrm{s}\right)$. 
These are slightly higher than findings previously reported in 1 case by Gauvain et al. ${ }^{[3]}$, which was $0.6\left(\times 10^{-3} \mathrm{~mm}^{2} / \mathrm{s}\right)$ and by Rumboldt et al. ${ }^{[6]}$ in two cases and theses readings were 0.55 and $0.63\left(10^{-}\right.$ $\left.{ }^{3} \mathrm{~mm}^{2} / \mathrm{s}\right)$. These differences are probably due to variation in measuring techniques. However, all still falling in the low ADC values characteristic of high-grade tumors.

AT/RT tumors have a population of rhabdoid cells and often additional variable amounts of other elements, such as neuroectodermal, epithelial, or mesenchymal histology. The tumors are always quite cellular, on the order of the other neoplasm most often in the differential diagnosis (i.e. medulloblastoma). Intracranially, these rare tumors are usually occuring in the cerebellum and are typically of heterogenous appearance on MR imaging [6].

In the studied cases of diffuse brain stem glioma, the signal intensity was hypointense on DWI compared to normal brain parenchyma denoting free diffusion.Some cases showed heterogeneous signal intensity in DWI and ADC map denoting high grade of anaplasia with ADC value ranging from $0.590 \times 10^{-3} \mathrm{~mm}^{2} / \mathrm{s}$ to $1.243 \times 10^{-3} \mathrm{~mm}^{2} / \mathrm{s}$ consistent with Lobel et al. This wide range of ADC values may because DPGs encompass a variety of tumors, ranging from WHO grade II to WHO grade IV tumors.Pilocytic Astrocytomas tend to arise in the cerebellum but could also occur in the brainstem.So, differentiation between them and DPG will be valuable $^{[14]}$.

ADC values and ratios were significantly different between pilocytic astrocytomas $\quad\left(1.69056 \pm 0.15 \times 10^{-3} \mathrm{~mm}^{2} / \mathrm{s}\right)$ (mean $\pm \mathrm{SD}$ ) and diffuse intrinsic pontine gliomas $\left(0.946 \pm 0.33 \times 10^{-3} \mathrm{~mm}^{2} / \mathrm{s}\right)$ with a $\mathrm{P}$ value $<0.01$ in the all three comparisons.There was no overlap in obtained measurements in case of mean ADC between these two tumors types.However, overlap occurred in the comparison of measurements of ratios.
Our optimal threshold for distinguishing medulloblastoma and JPA, ADC mean $0.847 \times 10^{-3} \mathrm{~mm}^{2} / \mathrm{s}$, was higher than the threshold used by Jaremko et al. [15] which was $0.800 \times 10^{-3} \mathrm{~mm}^{2} / \mathrm{s}$ as they used the minimum ADC value instead of the mean. Yet our results go with that of Rumboldt et al. ${ }^{[6]}$ likely because they also used ADC mean rather than minimum ADC.

The tumors included in our study were also sorted according to WHO grades as follows: 6 cases were grade I, 2 cases grade III and 16 cases were grade IV tumors.

According to our results, we found that the higher the tumor WHO grade, the lower the ADC in posterior fossa tumors.Therefore, we confirmed that diffusion restriction is rare in grade I tumors and common in grade IV tumors.

ADC values were significantly different between WHO I (low-grade tumors) $\left(1.69056 \pm 0.15 \times 10^{-3} \mathrm{~mm}^{2} / \mathrm{s}\right) \quad($ mean $\pm \mathrm{SD})$ and WHO IV (high- grade tumors) $\left(0.80352 \pm 0.23 \times 10^{-3} \mathrm{~mm}^{2} / \mathrm{s}\right),(\mathrm{P} \leq 0.001)$.

Measurement of ADC was no longer an experimental or time-consuming process. We were able to measure ADC rapidly with routine image-viewing software and no post processing was needed.

\section{Conclusion}

Quantitative evaluation of DW- MRI findings with generation of ADC values and ratios is a simple and readily available technique for the accurate evaluation and diagnosis of the majority of pediatric posterior fossa neoplasm. Solely relying on qualitative evaluation of DW images and ADC maps in differentiation of tumors of the posterior fossa might give rise to false results. Conventional MRI findings remain indispensable and results of diffusionweighted imaging should always be interpreted in conjunction with conventional MRI features. This is especially true of DIPGs where DWI plays a limited role in diagnosis of these tumors.

\section{References}

1. Poretti A and Meoded A (2012): Neuroimaging of pediatric posterior fossa tumors including review of the literature. Journal of Magnetic Resonance Imaging, 35:32-47. 
2. Jones DK (2011): Diffusion MRI: Theory, Methods, and Applications. 1st ed., Oxford University Press.

3. Gauvain KM, McKinstry RC, Mukherjee $P$ et al. (2001): Evaluating pediatric brain tumor cellularity with diffusion-tensor imaging. Am J Roentgenol., 177:44954

4. Moritani T., Ekholm S., Westesson $P$ (2005): Diffusion-Weighted MR Imaging of the Brain, $1^{\text {st }}$ ed., SpringerVerlag Berlin Heidelberg.

5. Zimmerman RD (2001): Is there a role for diffusion-weighted imaging in patients with brain tumors or is the "bloom off the rose"? AJNR Am J Neuroradiol., 22:1013-14

6. Rumboldt $\mathrm{Z}$, Camacho DL, Lake D et al. (2006): Apparent diffusion coefficients for differentiation of cerebellar tumors in children. Am J Neuroradiol., 27:1362-1369.

7. Stadnik TW, Chaskis C, Michotte A et al. (2001): Diffusion-weighted MR imaging of intracerebral masses: comparison with conventional MR imaging and histologic findings. Am J Neuroradiol., 22(5):969-76.

8. Bulakbasi N, Kocaoglu M, Ors F et al. (2003): Combination of single-voxel proton MR spectroscopy and apparent diffusion coefficient calculation in the evaluation of common brain tumors. AJNR Am J Neuroradiol., 24:225-233

9. Kan P, Liu JK, Hedlund G et al. (2006): The role of diffusion- weighted magnetic resonance imaging in pediatric brain tumors. Childs NervSyst., 22:1435-1439.

10. Yamasaki F, Kurisu K, Satoh K et al. (2005): Apparent diffusion coefficient of human brain tumors at MR imaging. Radiology, 235:985-91

11. Schneider JF, Confort-Gouny S, Viola A et al. (2007): Multiparametric differentiation of posterior fossa tumors in children using diffusion-weighted imaging and short echo-time 1H-MR spectroscopy. J MagnReson Imaging, 26:1390-98

12. Ellison D (2002): Classifying the medulloblastoma: insights from morphology and molecular genetics.

NeuropatholApplNeurobiol., 28:257-82

13. Cheng YC, Lirng JF, Chang FC et al. (2005): Neuroradiological findings in atypical teratoid/rhabdoid tumor of the central nervous system. Acta Radiol.,46:89-96

14. Sadighi Z, Slopis J (2013): (Pilocytic astrocytoma: a disease with evolving molecular heterogeneity. Unknown Journal, 28(5): 625-632.

15. Jaremko JL, Jans LB, Coleman LT et al. (2010): Value and limitations of diffusion-weighted imaging in grading and diagnosis of pediatric posterior fossa tumors. Am J Neuroradiol., 31:1613-1616. 